Pag.: 47 - 55

Artigo: Artigo original reportando investigação clínica ou básica DOI: https://doi.org/10.33194/rper.2021.v4.n1.163

\title{
A PESSOA SUBMETIDA A PRÓTESE PARCIAL DA ANCA: RELATO DE CASO
}

\section{THE PERSON UNDERGOING PARTIAL HIP PROSTHESIS: CASE REPORT}

\section{LA PERSONA QUE SE SOMETE A UNA PRÓTESIS PARCIAL DE CADERA: RELATO DE CASO}

\author{
Helena Teixeira ${ }^{(1)}$, Magali Palma ${ }^{(2)}$, João Vítor Vieira ${ }^{(3)}$, Maria José Bule(4),
}

(1) Centro Hospitalar Universitário do Algarve, Faro, Portugal; (2) Centro Hospitalar Universitário do Algarve, Faro, Portugal; (3) Instituto Politécnico de Beja Escola Superior de Saúde, Beja, Portugal; (4) Universidade de Évora, Évora, Portugal;

\section{Descritores}

\section{Enfermagem}

Prótese Parcial

Reabilitação

Enfermagem em Reabilitação

\section{Descriptors}

Hip Prothesis

Nursing

Rehabilitation

Rehabilitation Nursing

\section{Resumo}

Introdução: A fratura do fémur é um traumatismo que ocorre, frequentemente, em consequência de queda, sobretudo nos idosos. O enfermeiro especialista em enfermagem de reabilitacão é um profissional com competências que visam a restituição da funcionalidade, a minimização da incapacidade e dependência e o aumento da autonomia e da qualidade de vida.

Objetivo: Identificar os ganhos de um programa de reabilitação dirigido à pessoa submetida a prótese parcial da anca no internamento em Unidade de Convalescença.

Método: Estudo de caso descritivo de acordo com as guidelines da CAse REport (CARE). Após identificação das alterações músculo-esqueléticas, da funcionalidade e da definição dos diagnósticos, foi implementado um programa de reabilitação individualizado e dirigido aos objetivos e aos diagnósticos, destacando-se as intervenções de reeducação funcional motora e o treino de atividades de vida diárias.

Resultados: Comprovou-se o aumento da força muscular dos membros inferiores e superiores, o aumento da amplitude articular das articulacões dos membros inferiores, a melhoria no autocuidado andar com auxiliar de marcha e transferir-se e a diminuição do risco de queda.

Conclusões: Os resultados revelam efetividade das intervencões de enfermagem de reabilitação na pessoa submetida a prótese parcial da anca, ao nível da força e amplitude de movimentos e na execução e intensidade da marcha.

\section{Abstract}

Introduction: Femoral fracture is a trauma that often occurs as a result of a fall, especially in the elderly. The specialist nurse in rehabilitation nursing is a professional with skills that aim at restoring functionality, minimizing disability and dependence and increasing autonomy and quality of life.

Objective: To identify the gains from a rehabilitation program directed at the person who underwent partial hip replacement during admission to a Convalescence Unit.

Method: Descriptive case study according to the guidelines of CAse REport (CARE). After identifying the musculoskeletal changes, functionality and definition of diagnoses, an individualized rehabilitation program was implemented and aimed at objectives and diagnoses, highlighting interventions for functional motor reeducation and the training of daily life activities.

Results: An increase in the muscular strength of the lower and upper limbs, an increase in the joint amplitude of the joints of the lower limbs, an improvement in self-care walking with a walking aid and transfer, and a reduction in the risk of falling were proven.

Conclusions: The results reveal the effectiveness of rehabilitation nursing interventions in the person submitted to partial hip replacement, in terms of strength and range of motion and in the execution and intensity of gait.

\section{Resumen}

Introducción: La fractura de fémur es un trauma que a menudo ocurre como resultado de una caída, especialmente en los ancianos. La enfermera especialista en rehabilitación de enfermería es una profesional con habilidades que tienen como objetivo restaurar la funcionalidad, minimizar la discapacidad y la dependencia y aumentar la autonomía y la calidad de vida.

Objetivo: Identificar los beneficios de un programa de rehabilitación dirigido a la persona que se sometió a un reemplazo parcial de cadera durante su ingreso en una Unidad de Convalecencia. 
Método: Estudio de caso descriptivo de acuerdo con las directrices del CAse REport (CARE). Luego de identificar los cambios musculoesqueléticos, funcionalidad y definición de diagnosticos, se implementó un programa de rehabilitación individualizado y orientado a objetivos y diagnósticos, destacando las intervenciones para la reeducación motora funcional y el entrenamiento de las actividades de la vida diaria.

Resultados: Un aumento de la fuerza muscular de los miembros inferiores y superiores, un aumento de la amplitud articular de las articulaciones de los miembros inferiores, una mejora en el autocuidado de la marcha con andador y transfer, y una reducción del riesgo de Se han probado las caídas.

Conclusiones: Los resultados revelan la efectividad de las intervenciones de enfermería rehabilitadora en la persona sometida a artroplastia parcial de cadera, en términos de fuerza y amplitud de movimiento y en la ejecución e intensidad de la marcha.

\section{INTRODUÇÃO}

O envelhecimento é um fenómeno transversal nas sociedades ocidentais e congrega inúmeros determinantes. Em Portugal $51,3 \%$ da população com 16 e mais anos considerava ter um bom ou muito bom estado de saúde em 2020 , valor que diminui para $15,6 \%$ nas pessoas com 65 e mais anos ${ }^{(1)}$. A comparação dos dados com os valores dos países da União Europeia em 2019 coloca Portugal no grupo com perceção de saúde mais baixa ${ }^{(1)}$.

Assinalando-se nos últimos anos um crescente na perceção do estado de saúde em Portugal importa considerar que $73,8 \%$ da população com 65 e mais anos em 2020 tinham doenças crónicas ou um problema de saúde prolongado (i.e. duração igual ou superior a 6 meses) e destes $60,8 \%$ tinham limitação na realização de atividades, por problemas de saúde, com maior relevância nas mulheres ${ }^{(1)}$.

Os dados apresentados ilustram um grupo populacional que requer cuidados na gestão das situações crónicas e concomitantemente cuidados de apoio à realização das atividades de vida diária e de participação.

0 processo de envelhecimento fisiológico é frequentemente afetado por situações de doença, mas também por fenómenos acidentais como as quedas. As quedas acidentais originam fraturas e em Portugal no ano de 2018 foram a causa de 805 óbitos ${ }^{(2)}$. No Alentejo as cirurgias de artroplastia da anca aumentam desde 2019 e os dados disponiveis do primeiro quadrimestre de 2021 revelam um total de 56 cirurgias (Total de $2020=98)^{(3)}$. O risco de queda em idosos é multifatorial e a avaliação é a primeira etapa da prevenção ${ }^{(4)}$.

As quedas constituem uma importante causa de internamento, de morbilidade e de mortalidade na populacão com mais de 70 anos, pelo facto de, frequentemente, resultarem em lesões que causam perda de funcionalidade e, concomitantemente, perda de independência, o que leva a um decréscimo acentuado da qualidade de vida $(\mathrm{QV})^{(5)}$.

Resultante das quedas surgem um elevado número de fraturas da extremidade superior do fémur, sendo que, este incidente é mais frequente em mulheres em idade avançada, onde a queda ocorre, maioritariamente, no espaço interior da habitação. Este evento promove a perda de massa óssea, maior limitação física avaliada pela incapacidade de realizar atividades básicas da vida diária (ABVD) e instrumentais da vida diária (AIVD) e menor capacidade para deambular ${ }^{(6)}$. Um estudo realizado por Schuroff et al. (2013) ${ }^{(6)}$, que envolveu 93 pessoas submetidas a artroplastia parcial da anca cimentada, concluiu que em $20 \%$ da sua amostra existiu perda da capacidade de andar. Outro estudo, de Ono et al. $(2010)^{(6)}$, vem reforçar que a perda de capacidade para andar esteve presente em $33 \%$ da sua amostra. Estes dados, associados ao aumento da morbilidade e mortalidade decorrentes da fratura da extremidade superior do fémur nos idosos, trazem à luz a importância do papel do enfermeiro especialista em enfermagem de reabilitação (EEER) enquanto profissional com competências para a restituição da capacidade funcional da pessoa, minimização da incapacidade e da dependência e aumento da autonomia e qualidade de $\operatorname{vida}^{(6)}$.

O EEER detém competências para a implementação de programas de reabilitação na pessoa com alterações do aparelho locomotor que visem a promoção da maximização das capacidades e a redefinição de estratégias de modo a promover a reinserção social, familiar e profissional. Para além destes objetivos, os programas de reabilitação implementados pelo EEER podem assumir um papel importante na redução de taxas de reinternamento, na diminuição de complicações e no aumento da taxa de retorno da pessoa ao seu ambiente social e familiar ${ }^{(4,6)}$.

Neste âmbito, o objetivo geral deste estudo caracteriza-se por identificar os ganhos de um programa de reabilitação motora e treino de atividades de vida diária (AVD) à pessoa submetida a prótese parcial da anca, internada numa Unidade de Convalescença. Como objetivos específicos, pretende-se identificar as alteracões músculo-esqueléticas e de funcionalidade presentes na pessoa com recurso a escalas de avaliação; definir os principais diagnósticos de enfermagem de reabilitação relativos à pessoa submetida a prótese parcial da anca; implementar um programa de reabilitação individualizado para as necessidades e objetivos da pessoa; e por fim avaliar o impacto da implementação do programa de reeducação funcional motora e treino de AVD na pessoa, sensíveis aos cuidados de enfermagem de reabilitação.

\section{MATERIAL E MÉTODOS}

O estudo descritivo do tipo estudo de caso, consiste numa metodologia de investigação frequentemente utilizada para a compreensão de fenómenos $^{(7)}$ que se caracteriza pela capacidade de produzir evidência ${ }^{(8)}$. Tendo em conta estas considerações o presente estudo de caso foi elaborado de acordo com as guidelines da CAse REport (CARE) ${ }^{(9)}$.

O estudo descreve o caso de uma idosa com alteracões da mobilidade, força e equilíbrio postural em virtude de uma intervenção cirúrgica para colocação de prótese parcial da anca. A colheita de dados foi efetuada através da anamnese e consulta do processo clínico. 0 programa de enfermagem de reabilitação decorreu durante o mês de novembro de 2020 com duracão de 3 semanas, uma vez que o período temporal de internamento na unidade de convalescença é de 30 dias. Os resultados foram monitorizados, registados em função dos dias das sessões e encontram-se expostos no quadro 3. Importa referir que este estudo se trata de um caso único com abordagem holística.

O plano de reabilitação traçado para este estudo de caso teve a duração de 9 sessões divididas por três semanas, onde foram realizados 3 momentos de avaliacão: a avaliacão inicial no primeiro dia; a avaliacão intermédia no dia 11 de novembro de 2020 relativo à segunda semana; e a avaliacão final na última sessão, que teve lugar no dia 19 de novembro de 2020. A duração de cada sessão foi variável em função da capacidade e tolerância da pessoa, situando-se numa média de 1 hora de intervenção diária. 
A avaliação de enfermagem de reabilitação decorreu no dia 2 de novembro de 2020 através da recolha de dados do processo clínico, entrevista informal, observação direta e aplicação dos seguintes instrumentos de avaliação: Escala de Força de Medical Research Council para avaliacão da força muscular nos diferentes segmentos corporais; Escala de Ashworth modificada para avaliação da existência de espasticidade; Mini Mental State Examination (MMSE) para avaliar qual o estado de cognicão; Indice de Barthel para identificar o nível de dependência nas ABVD; Escala de Quedas de Morse para avaliar o risco de queda; Escala qualitativa para avaliar a dor e avaliação da amplitude dos diferentes segmentos articulares dos membros inferiores com recurso ao goniómetro ${ }^{(10)}$.

O estudo de caso foi realizado no âmbito de um estágio académico, aprovado pelas instituições envolvidas, em particular, um Centro Hospitalar e de uma Instituição de Ensino Superior do Sul de Portugal O projeto foi aprovado pela comissão de ética da Instituição de Ensino Superior $\left(N{ }^{\circ}\right.$ 0411/2020). A pessoa envolvida no estudo foi informada dos direitos relativos à sua participacão e foi obtido o seu consentimento livre e esclarecido antes do início da colheita de dados. Ressalva-se que foram respeitados todos os aspetos éticos, procurando-se cumprir as indicaç̃̃es expressas na Declaração de Helsínquia, do International Committee of Medical Journal Editors (ICMJE) e do Committee on Publication Ethics (COPE).

\section{APRESENTAÇÃO DO CASO}

\section{Anamnese}

O estudo reflete o caso de uma pessoa de 93 anos de idade, do sexo feminino, caucasiana, de nacionalidade portuguesa. É viúva e tem dois filhos a residir no estrangeiro e dois netos que prestam apoio direto. Tem o $4^{\circ}$ ano de escolaridade e trata-se de uma antiga costureira. Reside sozinha numa moradia no rés-do-chão com primeiro andar e era previamente autónoma nas AVD. Tem como antecedente pessoal dislipidemia, estando medicada para essa patologia.

A pessoa foi admitida no dia 7 de agosto de 2020 no Centro Hospitalar com o diagnóstico de fratura subcapital do fémur esquerdo decorrente de uma queda sofrida no domicílio. A senhora foi submetida a tratamento conservador de redução, estabilização e imobilização do membro inferior esquerdo até ao dia 14 de agosto de 2020, altura em que foi intervencionada por via de Hardinge para a colocação de uma prótese parcial da anca (PPA) à esquerda não cimentada. Durante o internamento, desenvolveu uma infeção da ferida cirúrgica tendo realizado antibioterapia, com resolucãa da infecão. Durante este internamento, a senhora desenvolveu uma úlcera por pressão de categoria IV no calcanhar esquerdo. Teve alta do internamento a 22 de outubro de $2020 \mathrm{com}$ a indicacão médica para efetuar levante e treino de marcha com carga completa no membro inferior esquerdo. Foi admitida na Unidade de Convalescença no mesmo dia, com data prevista de alta desta unidade a 20 de novembro 2020. Até à data de admissão nesta unidade foi submetida a intervenção de enfermagem de reabilitação durante o período pós-operatório, de aproximadamente dez dias.

\section{Avaliação de Enfermagem de Reabilitação}

Relativamente ao resultado obtido na avaliação da força muscular, através da aplicação da escala de Força de Medical Research Council, foi obtida forca $5 / 5$ em todos os segmentos corporais dos dois membros superiores. No membro inferior direito obteve-se forca $4 / 5$ em todos os segmentos e no membro inferior esquerdo força $1 / 5$ na flexão e extensão da coxa e da perna, e 2/5 flexão e extensão do pé. As limitações de força da senhora residiam principalmente ào nível dos membros inferiores e com maior incidência no membro inferior esquerdo, que foi submetido à intervenção cirúrgica. A nível dos membros superiores a senhora apresenta completa mobilidade e força mantida.

A Escala de Ashworth modificada foi também aplicada para avaliar o tónus muscular e determinar se perante a diminuição da força presente nos membros inferiores, se verifica a presença de espasticidade. Obteve-se grau 0 nos membros inferiores, concluindo-se que a senhora apresenta tónus normal.

O MMSE foi um instrumento de avaliação implementado, justificando-se o seu uso, pelo facto de, ao se aplicar questões como "Sabe onde se encontra?" ou "Que dia é hoje?", não se ter obtido resposta correta. Assim, considerou-se pertinente a aplicação deste instrumento para avaliar as funções cognitivas globais. O resultado obtido foi de 18/30, sendo que 30 é o máximo de total de pontos e revela diminuição do desempenho cognitivo ${ }^{(11)}$.

$O$ índice de Barthel caracteriza-se por um instrumento que permite avaliar o nível de independência da pessoa na realização de 10 AVD. A pontuação mínima é de 0 que corresponde à máxima dependência nas AVD e a pontuação de 100 diz respeito à independência total em todas as AVD avaliadas ${ }^{(10)}$. Após a aplicação deste instrumento a senhora obteve uma pontuação de 45 , preditor de dependência grave.

A Escala de Quedas de Morse foi também aplicada no sentido de perceber qual o risco de queda existente, tendo-se obtido a pontuação de 80 , indicativa de alto risco de queda presente.

A Escala Qualitativa da Dor foi a escala eleita para avaliar e quantificar a dor, pela sua fácil aplicação e compreensão por parte do utilizador.

A avaliação das amplitudes articulares dos membros inferiores foi realizada com recurso à goniometria.

\section{Diagnósticos de Enfermagem de Reabilitação}

Tendo em conta a avaliação realizada e de acordo com os défices e necessidades identificados foram nomeados os seguintes diagnósticos de enfermagem de reabilitação, baseados na linguagem da Classificação Internacional para a Prática de Enfermagem (CIPE $\left.{ }^{\circledR}\right)$ versão 2019(12) e no Padrão documental dos cuidados de enfermagem da especialidade de enfermagem de reabilitação ${ }^{(13)}$. Os défices cognitivos identificados pelo MMSE e as manifestações comportamentais que corroboravam o estado confusional condicionaram intervenções de enfermagem de reabilitação dirigidas aos autocuidados e por esse motivo não se definiram os respetivos diagnósticos de enfermagem.

Diagnóstico I: Movimento muscular diminuído;

Diagnóstico II: Andar com auxiliar de marcha comprometido;

Diagnóstico III: Capacidade para transferir-se comprometida; em grau moderado;

Diagnóstico IV: Dor presente nos membros inferiores;

Diagnóstico V: Confusão aguda presente;

Diagnóstico VI: Risco de queda presente, em grau elevado. 
Os diagnósticos anteriormente identificados permitem sustentar o programa de reeducação funcional motora e treino de AVD, apresentados de forma detalhada, no quadro 1.

Quadro 1 - Plano de Intervenção de Enfermagem de Reabilitação

\begin{tabular}{|c|c|c|}
\hline \multicolumn{3}{|c|}{ Plano de Intervenção de Enfermagem de Reabilitação } \\
\hline $\begin{array}{l}\text { Diagnóstico de } \\
\text { Enfermagem }\end{array}$ & $\begin{array}{l}\text { Resultado } \\
\text { Esperado }\end{array}$ & Intervenções de Enfermagem de Reabilitação \\
\hline \multirow[t]{2}{*}{$\begin{array}{c}\text { Movimento muscular } \\
\text { diminuído nos membros } \\
\text { inferiores }\end{array}$} & $\begin{array}{c}\text { Aumentar o } \\
\text { movimento } \\
\text { muscular e } \\
\text { amplitude } \\
\text { articular dos } \\
\text { membros } \\
\text { inferiores }\end{array}$ & $\begin{array}{l}\text { - } \quad \text { Avaliar a amplitude articular; } \\
\text { - } \quad \text { Executar exercícios isométricos dos músculos glúteos, quadricípites e abdominais; } \\
\text { - Executar exercícios isotónicos: mobilizações passivas do membro inferior esquerdo } \\
\text { movimentos de flexão/extensão, abdução/adução (até à linha média); } \\
\text { - } \quad \text { Realizar a técnica da ponte e rolamento no leito para o lado não afetado. }\end{array}$ \\
\hline & $\begin{array}{c}\text { Aumentar a força } \\
\text { dos membros } \\
\text { superiores e } \\
\text { inferiores }\end{array}$ & $\begin{array}{l}\text { - } \quad \text { Avaliar a força muscular; } \\
\text { - } \quad \text { Executar exercícios resistidos para os membros superiores com elástico de } \\
\text { resistência, bastão, pesos de } 1 \mathrm{~kg} \text { e pedaleira com resistência; } \\
\text { - } \quad \text { Realizar treino de levantar/sentar com apoio no espaldar; } \\
\text { - } \quad \text { Realizar treino de fortalecimento dos membros inferiores, em decúbito dorsal, } \\
\text { - } \quad \text { Treino de subir e descer escadas. }\end{array}$ \\
\hline $\begin{array}{l}\text { Andar com auxiliar de } \\
\text { marcha comprometido }\end{array}$ & $\begin{array}{c}\text { Marcha correta e } \\
\text { segura com apoio } \\
\text { de auxiliar de } \\
\text { marcha }\end{array}$ & $\begin{array}{l}\text { - } \\
\text { - } \quad \text { Avaliar e escolher o auxiliar de marcha mais adequado: andarilho; } \\
\text { - } \quad \text { Treinar andar com auxiliar de marcha; } \\
\text { - } \quad \text { Realizar treino de marcha a } 3 \text { pontos com andarilho (andarilho, perna operada, } \\
\text { - } \quad \text { Assistir e incentivar a pessoa a andar com o andarilho; } \\
\text { - } \quad \text { Realizar treino com pedaleira. }\end{array}$ \\
\hline $\begin{array}{c}\text { Capacidade para } \\
\text { transferir-se } \\
\text { comprometida em grau } \\
\text { moderado }\end{array}$ & $\begin{array}{c}\text { Transferência de } \\
\text { forma correta e } \\
\text { segura evitando a } \\
\text { flexão da } \\
\text { articulação coxo- } \\
\text { femoral esquerda } \\
\text { a mais de } 90^{\circ}\end{array}$ & $\begin{array}{l}\text { - } \quad \text { Assistir a pessoa a transferir-se pelo lado são; } \\
\text { - } \quad \text { Incentivar o alinhamento corporal; } \\
\text { - } \quad \text { Treinar o transferir-se: treinar rolamento para o lado da transferência; treinar carga } \\
\text { sobre o cotovelo; treinar sentar no leito; treinar equilíbrio e alinhamento corporal } \\
\text { sentado; treinar a assumir a posição ereta; treinar giro de } 180^{\circ} \text { com auxílio de } \\
\text { andarilho; treinar a sentar na cadeira com descida controlada evitando a flexão } \\
\text { superior a } 90^{\circ} \text { da articulação coxo-femoral esquerda. }\end{array}$ \\
\hline $\begin{array}{l}\text { Dor presente nos } \\
\text { membros inferiores }\end{array}$ & $\begin{array}{l}\text { Minimizar a dor } \\
\text { durante a } \\
\text { realização do } \\
\text { movimento } \\
\text { muscular }\end{array}$ & $\begin{array}{l}\text { - } \\
\text { - } \quad \text { Respaliar a presença de dor; } \\
\text { - } \quad \text { Não forçar o movimento na presença de dor; } \\
\text { - } \quad \text { Realizar massagem de conforto para redução da tensão muscular; } \\
\text { - } \quad \text { Respeitar os tempos de descanso. }\end{array}$ \\
\hline
\end{tabular}




\begin{tabular}{|c|c|c|}
\hline $\begin{array}{l}\text { Confusão aguda } \\
\text { presente }\end{array}$ & $\begin{array}{l}\text { Ativar uma } \\
\text { resposta } \\
\text { intencional e } \\
\text { apropriada }\end{array}$ & $\begin{array}{l}\text { - } \quad \text { Avaliar o estado mental; } \\
\text { - } \quad \text { Promover orientação no tempo e espaço; } \\
\text { - } \quad \text { Organizar o ambiente; } \\
\text { - } \quad \text { Orientar a pessoa; } \\
\text { - } \quad \text { Vestimular a memória; } \\
\text { - } \quad \text { Encorajar a participação na tomada de decisão; } \\
\text { - } \quad \text { Facilitar a orientação para a realidade. }\end{array}$ \\
\hline $\begin{array}{l}\text { Risco de queda } \\
\text { presente em grau } \\
\text { elevado }\end{array}$ & $\begin{array}{c}\text { Diminuir o risco e } \\
\text { evitar a queda }\end{array}$ & $\begin{array}{l}\text { - } \quad \text { Avaliar o risco de queda; } \\
\text { - } \quad \text { Gerir o ambiente; } \\
\text { - } \quad \text { Realizar treino de equilíbrio estático e dinâmico; } \\
\text { - } \quad \text { Realizar treino de técnicas de exercício muscular e articular; } \\
\text { - } \quad \text { Assistir na transferência; } \\
\text { - } \quad \text { Realizar treino de marcha com andarilho; } \\
\text { - } \quad \text { Vigiar atividade executada pela própria. }\end{array}$ \\
\hline
\end{tabular}

\section{RESULTADOS}

Os resultados obtidos na última sessão do programa de intervenção de enfermagem de reabilitação são apresentados no quadro 2.

A dependência nos autocuidados, avaliada pelo índice de Barthel, foi de 45 pontos, na avaliação inicial, e de 55 , na avaliação final, demonstrativa de dependência grave, com independência apenas na atividade alimentar-se. A evolução deveu-se à transição na atividade transferência cama-cadeira que inicialmente era realizada com grande ajuda e no final da intervenção apenas com pequena ajuda verbal e física e, na recuperacão da continência intestinal. Verificou-se igualmente uma redução no risco de queda através da avaliação pela escala de Quedas de Morse de 80 pontos para 65, manifestada na avaliação final, pela consciencialização das capacidades. No entanto, como referido anteriormente, a intervenção nos autocuidados não foi exequível face aos défices cognitivos flutuantes manifestados durante o período de contacto.

Quadro 2 - Resultados obtidos na última sessão

\begin{tabular}{|c|c|c|}
\hline \multicolumn{3}{|c|}{ Resultados obtidos na última sessão } \\
\hline $\begin{array}{c}\text { Frequência (série e } \\
\text { repetições) ou distância } \\
\text { percorrida na última sessão }\end{array}$ & Tipo de exercício & Resultados \\
\hline 1 série de 12 repetições & 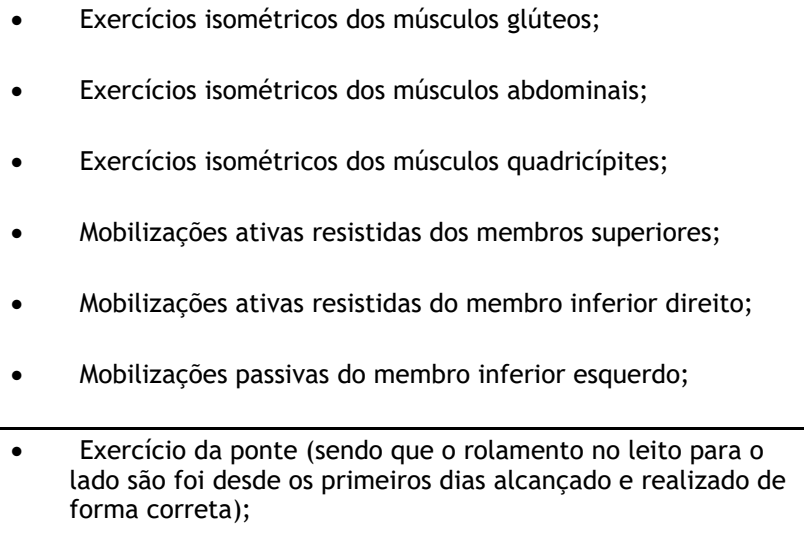 & $\begin{array}{l}\text { Verificou-se um aumento global } \\
\text { das amplitudes articulares, } \\
\text { concretamente, um aumento } \\
\text { médio de } 5,6^{\circ} \text { nos segmentos } \\
\text { osteoarticulares do membro } \\
\text { inferior esquerdo, e um aumento } \\
\text { médio de } 4,5^{\circ} \text { nos segmentos } \\
\text { osteoarticulares do membro } \\
\text { inferior direito. } \\
\text { Obteve-se uma transição na } \\
\text { escala de força } M R C \text {, do membro } \\
\text { inferior esquerdo, de } 1 / 5 \text { para } \\
2 / 5 \text { na flexão e extensão da coxa } \\
\text { e da perna, e de } 2 / 5 \text { para } 3 / 5 \text { na } \\
\text { flexão e extensão do pé. }\end{array}$ \\
\hline
\end{tabular}




\begin{tabular}{|c|c|c|c|}
\hline \multirow[t]{2}{*}{2 séries de 10 repetições } & \multirow[t]{2}{*}{$\begin{array}{l}\text { - } \quad \text { Exercícios } \\
\text { resistidos dos } \\
\text { membros } \\
\text { superiores }\end{array}$} & 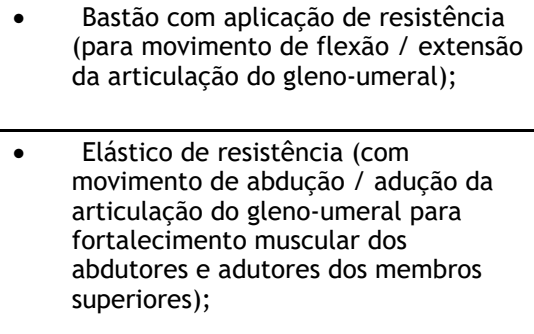 & \multirow[t]{2}{*}{$\begin{array}{l}\text { - } \quad \text { Verificou-se, desde o início do } \\
\text { programa, uma força muscular } \\
\text { nos membros superiores, avaliada } \\
\text { pela escala de força MRC, de } 5 / 5 \\
\text { em todos os segmentos } \\
\text { musculares. No entanto, } \\
\text { considera-se que se alcançou um } \\
\text { aumento da resistência muscular } \\
\text { pela concretização de um maior } \\
\text { número de séries e repetições } \\
\text { (evolução registada no quadro 3). }\end{array}$} \\
\hline & & $\begin{array}{l}\text { - Peso de } 1 \mathrm{~kg} \text { para fortalecimento de } \\
\text { bicípite braquial; }\end{array}$ & \\
\hline 1 série de 9 repetições & \multicolumn{2}{|c|}{$\begin{array}{l}\text { Treino com pedaleira para mobilização da articulação do } \\
\text { joelho em posição sentada com altura suficiente para evitar a } \\
\text { flexão da articulação coxo-femoral superior a } 90^{\circ} ;\end{array}$} & \multirow{3}{*}{$\begin{array}{l}\text { - Alcançou-se uma marcha segura } \\
\text { com apoio de andarilho por } \\
\text { distâncias que evoluíram de } 15 \\
\text { para } 60 \text { metros, na última } \\
\text { avaliação. Desta forma garantiu- } \\
\text { se uma quadruplicação na } \\
\text { distância percorrida e a melhoria } \\
\text { no autocuidado andar com } \\
\text { auxiliar de marcha. }\end{array}$} \\
\hline 60 metros & \multicolumn{2}{|c|}{$\begin{array}{l}\text { Treino de marcha com andarilho com distância de } 60 \text { metros } \\
\text { (30 metros, descanso, } 30 \text { metros); }\end{array}$} & \\
\hline-- & \multicolumn{2}{|c|}{$\begin{array}{l}\text { - Treino de subir e descer escadas, apesar de constar nos } \\
\text { objetivos do programa de intervenção de reabilitação e de } \\
\text { ter sido realizada a sua instrução, não foi possível de realizar } \\
\text { devido ao elevado grau de dependência nesta atividade. }\end{array}$} & \\
\hline
\end{tabular}

\section{CRONOGRAMA}

\begin{tabular}{|c|c|c|c|c|c|c|c|c|c|}
\hline & \multicolumn{3}{|c|}{$1^{\text {a }}$ semana } & \multicolumn{3}{|c|}{$2^{\mathrm{a}}$ semana } & \multicolumn{3}{|c|}{$3^{\text {a }}$ semana } \\
\hline & $\begin{array}{c}\text { Sessão } \\
1\end{array}$ & $\begin{array}{c}\text { Sessão } \\
2\end{array}$ & $\begin{array}{c}\text { Sessão } \\
3\end{array}$ & $\begin{array}{c}\text { Sessão } \\
4\end{array}$ & $\begin{array}{l}\text { Sessão } \\
5\end{array}$ & $\begin{array}{c}\text { Sessão } \\
6\end{array}$ & $\begin{array}{c}\text { Sessão } \\
7\end{array}$ & $\begin{array}{c}\text { Sessão } \\
8\end{array}$ & $\begin{array}{c}\text { Sessão } \\
9\end{array}$ \\
\hline \multicolumn{10}{|l|}{$\begin{array}{l}\text { Avaliação de } \\
\text { ER }\end{array}$} \\
\hline $\begin{array}{l}\text { Exercícios } \\
\text { isométricos } \\
\text { (glúteos, } \\
\text { abdominais e } \\
\text { quadricípites) }\end{array}$ & * & * & $\dagger$ & $\dagger$ & $\ddagger$ & $\neq$ & $\dagger$ & $\S$ & $\S$ \\
\hline $\begin{array}{l}\text { Exercícios } \\
\text { isotónicos:MAA } \\
\text { MI drt., MP MI } \\
\text { esq, MAR MS }\end{array}$ & * & * & $\dagger$ & $\dagger$ & $\ddagger$ & $\S$ & $\dagger$ & $\S$ & $\S$ \\
\hline $\begin{array}{l}\text { Técnica da } \\
\text { ponte e } \\
\text { rolamento no } \\
\text { leito para o } \\
\text { lado são }\end{array}$ & & & * & * & $\dagger$ & $\ddagger$ & * & $\dagger$ & $\neq$ \\
\hline $\begin{array}{l}\text { Treino de } \\
\text { força dos MS } \\
\text { (com bastão, } \\
\text { elástico e peso } \\
\text { de } 1 \mathrm{~kg} \text { ) }\end{array}$ & & & $\ddagger$ & $\ddagger$ & $\ddagger$ & $\S$ & $\S$ & $\S$ & $\S$ \\
\hline $\begin{array}{l}\text { Treino de } \\
\text { equilibrio } \\
\text { sentado e } \\
\text { treino de } \\
\text { marcha com } \\
\text { AM }\end{array}$ & & & II & 11 & II & १ & ** & ๆๆ & ๆๆ \\
\hline $\begin{array}{l}\text { Treino de } \\
\text { levantar } \\
\text { /sentar com } \\
\text { ajuda }\end{array}$ & & & $\dagger$ & ** & ** & $\dagger$ & ** & * & \\
\hline $\begin{array}{l}\text { Treino de } \\
\text { pedaleira }\end{array}$ & & & & & & * & ** & *** & $\dagger$ \\
\hline $\begin{array}{l}\text { Treino de } \\
\text { subir/ descer } \\
\text { escadas }\end{array}$ & & & & & & & & & ** \\
\hline
\end{tabular}

Quadro 3 - Cronograma do Plano de Intervenção de Reeducação Funcional Motora 
Legenda:

Avaliação Enfermagem de Reabilitação (MMSE, Escala MRC, Escala de Ashwoth modificada, Índice de Barthel, Escala de Morse, Escala qualitativa da dor, Goniometria)

Intervenções realizadas no leito

Intervenções realizadas em ortostatismo

\author{
* 1 série $<5$ repetições \\ † 1 série $\geq 5$ - 10 repetições \\ ¥ 1 série $\geq 10$ repetições \\ $\S 2$ séries - 10 repetições \\ | | Distância < 30 metros \\ ๆ Distância $\geq 30$ metros \\ ** Não realizado
}

MAA - Mobilizações Ativas-Assistidas / MP - Mobilizações Passivas / MAR - Mobilizações Ativas Resistidas / MI - Membro Inferior / MS - Membro Superior / AM - Auxiliar de Marcha.

\title{
DISCUSSÃO
}

Perante o caso clínico de prótese parcial da anca devido a fratura subcapital do fémur esquerdo por queda, foi delineado um programa de enfermagem de reabilitação fundamentado em evidência científica e direcionado para a condição clínica da pessoa, permitindo uma abordagem individual e holística.

0 método exposto e os resultados alcançados permitem demostrar que os cuidados especializados de enfermagem de reabilitação desempenham um papel preponderante na recuperação da funcionalidade da pessoa com alterações músculo-esqueléticas.

O programa de reabilitação no pós-operatório deverá incidir sobre mobilização articular, fortalecimento muscular, prevenção de luxação, treino de verticalização e marcha e treino de AVD's, pelo que após a avaliação da senhora foram identificados os focos e diagnósticos de intervenção e delineado um programa de reabilitação assente em: reeducação funcional motora, treino de AVD, fortalecimento muscular, aumento de amplitude articular, treino de marcha com auxiliar de marcha, prevenção de luxação da anca, prevenção de queda, gestão da dor e confusão(14). Ainda que a intervenção não tenha sido implementada num período de pós-operatório propriamente dito, face à situação de fragilidade da pessoa e, sobretudo, aos diagnósticos identificados, particularmente os diagnósticos diretamente relacionados com as alteraçõ̃es músculo-esqueléticas e com as alterações de funcionalidade, privilegiou-se a implementação de um programa que contemplasse intervenções de reeducação funcional motora dirigidas às alterações provocadas pela realização da prótese parcial da anca e ao treino de actividades de vida diárias.

A implementação do plano de intervenção de enfermagem de reabilitação viabilizou ganhos em termos de mobilidade e de movimento muscular, nomeadamente, a nível dos membros inferiores e superiores, que se constata pelo aumento do número de repetições e séries de exercícios realizados e pela transicão na escala de força MRC, sobretudo o membro inferior esquerdo, de $1 / 5$ na flexão e extensão da coxa e perna para $2 / 5$, e de $2 / 5$ para $3 / 5$ na flexão e extensão do pé. Adicionalmente, comprovou-se uma duplicação na distância percorrida com treino de marcha, para além das melhorias a nível das amplitudes articulares, verificando-se um aumento médio de $5,6^{\circ}$ no membro inferior esquerdo e de $4,5^{\circ}$ no membro inferior direito. Na avaliação final obtiveram-se também melhores resultados nas escalas de Morse, Braden e Mini Mental State Examination.

Em virtude do estado confusional da pessoa, que frequentemente limitou a realização dos exercícios, estas melhorias foram obtidas de forma gradual e progressiva. A confusão aguda, entre muitas causas, pode verificar-se em situações não orgânicas, como alterações súbitas no ambiente da pessoa idosa, nomeadamente a hospitalização, que pode estar na origem deste quadro. O declínio cognitivo constitui um fator que pode prejudicar a capacidade de andar, o que torna imperativo a adaptação do programa de reabilitação à individualidade de

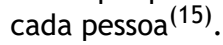

O incentivo para executar as intervencões planeadas foi constante e a presença de dor nos membros inferiores, em particular no membro inferior esquerdo, foi limitante em determinados exercícios, verificando-se a necessidade de selecionar as intervencões a implementar com respeito permanente pela capacidade funcional, tolerância, nível de cansaço e dor. Estes aspetos conduziram a uma adaptabilidade do programa de reabilitacão, o que se considera habitual e frequente, uma vez que os ensinos e os treinos devem ser adaptados à condição do idoso e às alterações fisiológicas inerentes a este facto. Destaca-se que a pessoa tendencionalmente irá sofrer um défice de força durante aproximadamente dois anos após a cirurgia, devido a atrofia dos músculos periarticulares da anca dolorosa ${ }^{(14,16)}$.

Apesar das limitações inerentes à condição clínica, é possível afirmar que as intervenções do EEER se revelaram essenciais para alcançar os objetivos propostos e para a melhoria global da mobilidade, amplitude articular, realização de AVD e redução do risco de queda.

$\mathrm{O}$ treino de AVD consiste numa intervenção imprescindível do EEER, dado que possibilita a aquisição da máxima funcionalidade e qualidade de vida. Este treino permite desenvolver o aumento da força muscular, amplitude do movimento articular, melhorar o equilíbrio e aumentar a capacidade de marcha, o que promove e potencia a independência no autocuidado ${ }^{(17)}$. 0 encorajamento e o sentimento de segurança, o reforço positivo e a valorização das conquistas constituem chaves de sucesso para um programa de reabilitação eficaz ${ }^{(18)}$. Refira-se ainda que, atualmente, a evidência científica defende que o treino será tão eficaz quanto mais oportunidades existirem para o realizar ao longo do dia, sendo mais benéfico um treino de curta duração e várias vezes ao dia, em detrimento a um treino prolongado uma única vez ao $\operatorname{dia}^{(18)}$. 
Como limitações deste estudo de caso identifica-se a idade e as flutuações do estado cognitivo devido a confusão aguda, frequentemente responsáveis pela menor colaboração na realização de determinados exercícios. Salienta-se ainda, como limitação ao estudo, o período de 15 dias de isolamento profilático associado à Pandemia da Covid-19, aquando da admissão da senhora na Unidade de Convalescenca, que restringiu a possibilidade de executar um maior número de intervenç̃̃es e de realizar treinos no ginásio. Face a esta limitação, considera-se que seria pertinente alargar o tempo disponível para a implementação do programa de enfermagem de reabilitação, com vista à obtenção de resultados mais consistentes.

\section{CONCLUSÃO}

Este estudo de caso permitiu destacar a importância e efetividade das intervenções de enfermagem de reabilitação implementadas pelo EEER na pessoa submetida a prótese parcial da anca, ao nível da funcionalidade motora e da realização de AVD. Ao longo da implementação do programa de intervenção foram necessários pontuais ajustes nos objetivos a alcancar, em virtude da capacidade e da tolerância da pessoa e, sobretudo, da sua cooperação e participação nas atividades propostas. Os instrumentos de avaliação selecionados constituíram um auxílio imprescindível para a definição dos diagnósticos de enfermagem e viabilizaram a monitorização e a avaliação das atividades e dos resultados obtidos, assim como a individualização do programa que promoveu uma abordagem holística. A diminuta eficácia das intervenções dirigidas à dependência nos autocuidados emerge como fator condicionante da recuperação funcional e esteve sempre associado aos défices cognitivos que, embora flutuantes, não permitiram as aquisições necessárias.

A evolução da condicão clínica da pessoa foi, de uma forma global, notória, ainda que as melhorias se tenham revelado mais evidentes em determinados diagnósticos, particularmente, na melhoria do movimento muscular, no aumento de força dos musculares dos membros superiores e inferiores, no aumento das amplitudes articulares e no aumento da qualidade e da distẩncia percorrida com auxiliar de marcha. Estes dados permitem justificar a importância da intervenção do EEER junto da pessoa com alterações orto-traumatológicas através da implementação de um programa de reeducação funcional motora e treino de AVD personalizado e adaptado, proporcionando uma melhoria da funcionalidade e consequente qualidade de vida.

\section{DIVULGAÇÕES ÉTICAS}

Contribuição do(s) autor(es):

Conceptualização: HT, MP, JVV;

Metodologia: HT, JVV; MJB;

Validação: HT, MP, JVV; MJB;

Análise formal: HT, JVV;

Investigação: HT, MP, JVV;

Tratamento de dados: HT, MP, JVV;

Preparação do rascunho original: HT, JVV; MJB;

Redação e edição: HT, MP, JVV; MJB;

Revisão: HT, MP, JVV; MJB.

Todos os autores leram e concordaram com a versão publicada do manuscrito.

Financiamento:

Este trabalho não recebeu nenhuma contribuição financeira ou bolsa.

Comissão de Ética:

Estudo autorizado pela Comissão de Ética da Instituição de Ensino Superior (0411/2020).

Declaração de consentimento informado:

O consentimento informado por escrito para publicar este trabalho foi obtido do participante.

Conflitos de interesse:

Os autores não declaram nenhum conflito de interesses.

Proveniência e revisão por pares:

Não comissionado; revisto externamente por pares. 


\section{Referências}

1. Instituto Nacional de Estatística. Estatísticas da Saúde-2019. Lisboa: INE; 2021. Disponível em:

https: / / www.ine.pt/xportal/xmain?xpid=INE\&xpgid=ine_publicacoes\&PUBLICACOESpub_boui=257483090\&PUBLICACOESmodo=2

2. Portuguese Arthroplasty Register. Hospitais/Estatísticas 2021. Disponível em:

http: //www.rpa.spot.pt/Main-Sections/Hospitals.aspx?Arsld=4.

3. Instituto Nacional de Estatística. Causas de morte 2015. Lisboa: Instituto Nacional de Estatística; 2017.

4. Fernandes JB, Sá MCL, Nabais, ASC. Intervenções do Enfermeiro de Reabilitação que Previnem a Ocorrência de Quedas na Pessoa Idosa: Revisão Scoping. RPER. 2020; 3(1): 57-63. Disponível em: 10.33194/rper.2020.v3.n1.7.5761

5. Direção Geral da Saúde. Programa nacional de prevenção de acidentes 2010-2016; 2010 [Consultado a 22/11/2020]. Disponível em: https://www.dgs.pt/ficheiros-de-upload-3/dast-programa-nacional-de-prevencao-de-acidentes-pdf.aspx.

6. Sousa L., Carvalho ML. Pessoa com Fratura da Extremidade Superior do Fémur. In Marques-Vieira C, Sousa L, Eds. Cuidados de enfermagem de reabilitação à pessoa ao longo da vida. Loures: Lusodidacta; 2016. p. 421-431.

7. Andrade SR, Ruoff AB, Piccoli T, Schmitt MD, Ferreira A, Xavier AC. O estudo de caso como método de pesquisa em enfermagem: uma revisão integrativa. Texto Contexto Enferm. 2017; 26(4): 1-12. Disponível em: 10.1590/0104-07072017005360016.

8. Raposo P, Sousa L. Intervenção do Enfermeiro Especialista em Reabilitação na Dispneia da Pessoa com COVID-19: Relato de Caso. RPER. 2020; 3(s2). 5-15. Disponível em: 10.33194/rper.2020.v3.s2.1.5773.

9. Riley DS, Barber MS, Kienle GS, Aronson JK, Schoen-Angerer T, Tugwell P ... Gagnier JJ. CARE guidelines for case reports: explanation and elaboration document. J Clin Epidemiol. 2017; 89: 218-235. Disponível em: 10.1016/j.jclinepi.2017.04.026.

10. Ordem dos Enfermeiros. Enfermagem de Reabilitação - Instrumentos de recolha de dados para a documentação dos Cuidados Especializados em Enfermagem de Reabilitação. 2016. Disponível em:

https: / / www.ordemenfermeiros.pt/arquivo/colegios/Documents/2017/InstRecolhaDadosDocumentacaoCuidEnfReabilitacao_Final_2017.pdf.

11. Morgado J, Rocha CS, Maruta C, Guerreiro M, Martins IP. Novos valores normativos do Mini-Mental State Examination. Sinapse. 2009. P. 916. Disponível em:

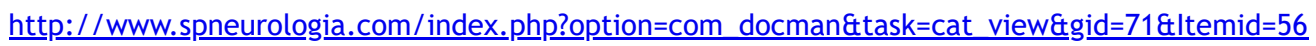

12. International Council of Nurses. ICNP Browser. 2019. Disponivel em: https://www.icn.ch/what-we-do/projects/ehealth-icnptm/icnpbrowser

13. Ordem dos Enfermeiros. Padrão documental dos cuidados de enfermagem da especialidade de enfermagem de reabilitação. Porto: Mesa do Colégio da Especialidade de Enfermagem de Reabilitação; 2015. Disponível em:

https://www.ordemenfermeiros.pt/arquivo/colegios/Documents/2015/MCEER_Assembleia/PadraoDocumental_EER.pdf.

14. Parente C, Ângelo M, Tinoco A, Rocha E, Brandão D, Cristina M, Céu M. Artroplastia da Anca. In Cruz A, Conceição V, Oliveira L, Eds. Enfermagem em Ortotraumatologia. Coimbra: Formasau; 2009. p. 129-164.

15. Varanda E, Rodrigues C. Avaliação da Pessoa com Alterações da Função Cognitiva: Avaliar para Reeducar e Readaptar. In Marques-Vieira C, Sousa L, Eds. Cuidados de enfermagem de reabilitação à pessoa ao longo da vida. Loures: Lusodidacta; 2016. p. 145-157.

16. Chainça L, Rebelo O, Almeida T. Fraturas do Terço Superior do Fémur. In Cruz A, Conceição V, Oliveira L, Eds. Enfermagem em Ortotraumatologia. Coimbra: Formasau; 2009. p. 186-203.

17. Vigia C, Ferreira C, Sousa L. Treino de Atividades de Vida Diária. In Marques-Vieira C, Sousa L, Eds. Cuidados de enfermagem de reabilitação à pessoa ao longo da vida. Loures: Lusodidacta; 2016. p. 351-363.

18. Marques-Vieira C, Caldas A. A Relevância do Andar: Reabilitar a Pessoa Com Andar Comprometido. In Marques-Vieira C, Sousa L, Eds. Cuidados de enfermagem de reabilitação à pessoa ao longo da vida. Loures: Lusodidacta; 2016. p. 547-557.

Este trabalho encontra-se publicado com a Licença Internacional Creative Commons Atribuição-NãoComercial-SemDerivações 4.0.

Direitos de Autor (c) 2021 Revista Portuguesa de Enfermagem de Reabilitação 